ITEP-TH-17/01

\title{
Possible Pseudogap Phase in QCD
}

\author{
K. Zarembo \\ Department of Physics and Astronomy \\ and Pacific Institute for the Mathematical Sciences \\ University of British Columbia \\ 6224 Agricultural Road, Vancouver, B.C. Canada V6T $1 Z 1$ \\ and \\ Institute of Theoretical and Experimental Physics, \\ B. Cheremushkinskaya 25, 117259 Moscow, Russia \\ E-mail: zarembo@physics.ubc.ca
}

\begin{abstract}
Thermal pion fluctuations, in principle, can completely disorder the phase of the quark condensate and thus restore chiral symmetry. If this happens before the quark condensate melts, strongly-interacting matter will be in the pseudogap state just above the chiral phase transition. The quark condensate does not vanish locally and quarks acquire constituent masses in the pseudogap phase, despite chiral symmetry is restored.
\end{abstract}


The physics of light hadrons is to a large extent controlled by the approximate chiral symmetry of QCD. The order parameter of the chiral symmetry, the quark condensate $\langle\bar{\psi} \psi\rangle$, acquires a non-zero expectation value in the QCD vacuum and chiral symmetry appears spontaneously broken. Chiral symmetry breaking gives quarks constituent masses of order of 350-400 Mev and thus sets the scale of hadron masses. Pions arise as lowenergy, pseudo-Goldstone excitations of the chiral condensate. If strongly-interacting matter is heated to the temperature that exceeds a critical value of 150-200 Mev, chiral symmetry gets restored. Usually, the restoration of chiral symmetry is associated with melting of the quark condensate. I will discuss a less familiar mechanism of symmetry restoration by phase decoherence which, if realized, implies the existence of an intermediate phase, similar to the pseudogap phase of high- $T_{c}$ superconductors, the analogy with which I will extensively use. In the pseudogap phase, quarks still condense and acquire constituent masses, but chiral symmetry is not broken because the phase of the condensate is completely disordered. The potential relevance of the pseudogap phenomenon to QCD was pointed out by Babaev and Kleinert [1, 2], who examined low-dimensional toy models of chiral symmetry breaking

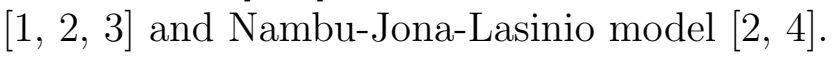

A continuous symmetry associated with the complex order parameter $\Psi=\rho \mathrm{e}^{i \varphi}$ is usually restored when the free energy of the symmetry-breaking state with non-vanishing condensate $\rho \neq 0$ starts to exceed the free energy of the symmetric state with $\rho=0$. However, phase decoherence can restore the symmetry even if $\rho \neq 0$. When the phase of the condensate is completely disordered: $\left\langle\mathrm{e}^{i \varphi}\right\rangle=0$, the expectation value of the order parameter, apparently, vanishes: $\langle\Psi\rangle=0$. There is a growing evidence that such a mechanism is realized in some high- $T_{c}$ superconductors [5, 6], whose normal, non-superconducting state possesses many features characteristic of superconductivity. Most notably, the energy gap does not close above the point at which superconductivity is destroyed and gradually disappears only at much higher temperature. A theoretical explanation of the pseudogap phenomenon [5] relies on the essentially two-dimensional nature of high- $T_{c}$ superconductivity. In two dimensions, topological phase fluctuations of the superconducting condensate (vortices) are extremely important. Depending on the temperature and on the phase stiffness (the energy cost of phase fluctuations), vortices either are bound in pairs or form a plasma. These two phases are separated by Berezinsky-Kosterlitz-Thouless (BKT) transition [7]. In high$T_{c}$ superconductors, the temperature of BKT transition is lower than the temperature at which the condensate of Cooper pairs melts and superconducting gap accordingly shrinks. The superconductivity is then destroyed by vortices that unbind at the BKT transition and completely disorder the condensate's phase.

In QCD, the chiral order parameter is an $N_{f} \times N_{f}$ matrix ( $N_{f}$ is the number of light quark species): $\Sigma_{f f^{\prime}}=\bar{\psi}_{f} \psi_{f^{\prime}}$. Its vacuum expectation value is diagonal because light quarks have small current masses which align the condensate in the particular direction. Throughout this paper I will discuss the chiral limit and neglect quark masses. The coherent fluctuations of the condensate's phase cost no energy in this approximation and correspond to Goldstone modes of the broken chiral symmetry. The chiral phase of the quark condensate is an unitary $N_{f} \times N_{f}$ matrix:

$$
\mathrm{e}^{i \gamma^{5} \lambda^{a} \pi^{a}}=\frac{1-\gamma^{5}}{2} U+\frac{1+\gamma^{5}}{2} U^{\dagger}
$$




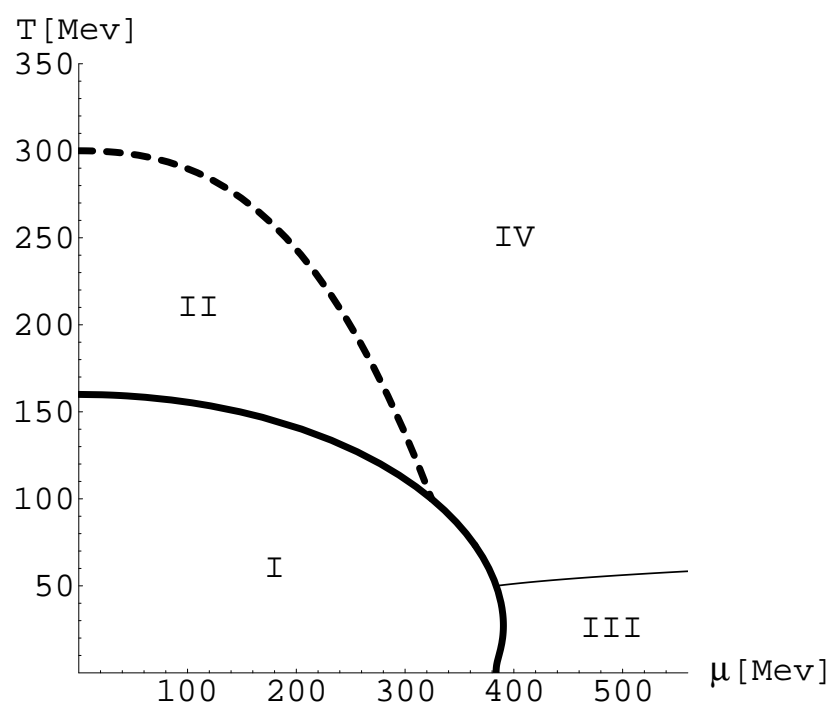

Figure 1: QCD phase diagram: I - hadron phase, II - pseudogap phase, III - color superconducting phases, IV - quark-gluon plasma.

where $\lambda^{a}$ are $S U\left(N_{f}\right)$ generators and $\pi^{a}$ are Goldstone fields associated with pions.

The low-energy dynamics of pions is described by the chiral Lagrangian:

$$
\mathcal{L}_{\chi}=\frac{F_{\pi}^{2}}{4} \operatorname{tr} \partial_{\mu} U^{\dagger} \partial^{\mu} U
$$

where $F_{\pi}=93 \mathrm{Mev}$ is the pion decay constant. The classical thermodynamics of the nonlinear sigma model has been extensively studied by Monte Carlo simulations [8, 9] and, indeed, the phase transition associated with disordering of the chiral field has been found in numerical simulations: $\langle\operatorname{tr} U\rangle \neq 0$ below the critical point and $\langle\operatorname{tr} U\rangle=0$ above. The large- $N$ analysis [10] suggests that the phase transition does not disappear if one goes from classical thermodynamics to quantum. Since

$$
\langle\bar{\psi} \psi\rangle=\left\langle|\Sigma|\left(\operatorname{tr} U+\operatorname{tr} U^{\dagger}\right)\right\rangle
$$

the chiral condensate turns to zero when $\langle\operatorname{tr} U\rangle=0$ and chiral symmetry gets restored. The question is whether the temperature of pion disordering is lower or higher than the temperature at which the quark condensate melts. If it is lower, as suggested by the smallness of the pion decay constant (the analog of phase stiffness in QCD), then the pseudogap phase exists in the intermediate range of temperatures. The results of Refs. [11, 10] seem to support the hypothesis that the chiral phase transition is essentially driven by angular fluctuations of the quark condensate.

If the chiral transition is caused by pion decoherence, the phase diagram of QCD will look as shown in Fig. 1, with the pseudogap phase sandwiched between the hadron and the quark-gluon plasma phases. It should be mentioned that no symmetry and no order parameter can distinguish the pseudogap phase from the quark-gluon plasma, since the separation of 
the quark condensate in the phase and the modulus only makes sense within the low-energy approximation, when phase fluctuations are sufficiently light compared to all other modes. The pseudogap phase cannot be separated from quark-gluon plasma by a phase transition, inasmuch as there is no phase transition between the pseudogap state and the normal state in high- $T_{c}$ superconductors. The dashed line in Fig. 1 thus denotes a smooth crossover, which can be rather broad. The distinctive feature of the pseudogap phenomenon is that the symmetry restoration affects only the Goldstone modes, while parameters associated with other excitations are continuous or almost continuous across the phase transition. In particular, masses of all excitations in the pseudogap phase, except for pions, will be determined by constituent quark mass and thus will be rather large. If there is no pseudogap phase and the constituent quark mass disappears above the chiral transition, masses of non-Goldstone modes are expected to drop at the critical temperature.

A particular mechanism that can lead to pion decoherence is disordering of the chiral condensate by baryons [9, 12]. The idea behind this mechanism closely follows the analogy with high- $T_{c}$ superconductivity. In the scenario proposed in [9, 12], baryons disorder the chiral condensate in the same way vortices disorder the phase of the superconducting gap in two dimensions. The key point is that baryons can be associated with topological excitations of the chiral field [13. Inside the baryon, $U(x)$ winds around an $S U(2)$ subgroup of $S U\left(N_{f}\right)$. Therefore, in a sufficiently dense random ensemble of baryons and anti-baryons, the chiral field is randomly distributed over $S U\left(N_{f}\right)$, which results in $\langle\operatorname{tr} U\rangle=0$. Numerical simulations of 3D sigma model [9] indicate that this picture is probably correct. In particular, baryon susceptibility undergoes a dramatic rise in the vicinity of the phase transition [9]. It was independently observed [12] the thermal density of an ideal baryon gas is comparatively large already at $T \sim 150-200 \mathrm{Mev}$, despite a small Boltzmann factor associated with large baryon masses. The latter is compensated by a large entropy due to a large number of baryon resonances. The phase transition in the non-linear sigma model from this point of view resembles the BKT transition [12]. The pseudogap phase is then analogous to the high-temperature, plasma phase of 2D XY model, in which vortices are liberated and all correlations are screened. Similarly, there is no pions in the pseudogap phase and all excitations of the chiral field must be massive.

The whole idea of the pseudogap mechanism relies on the assumption that the low-energy approximation is still accurate near the chiral phase transition. Therefore, the effective chiral Lagrangian (1) should still make sense in the pseudogap phase, though chiral perturbation theory, at least in its straightforward implementation, should break down. Since the linear and the non-linear sigma models share the same symmetries and belong to the same universality class, the order of the transition is determined by universality arguments of and depends on $N_{f}$ [14]. For realistic quark masses, Monte Carlo simulations indicate [15] that the transition becomes sharp but smooth crossover.

The phase transition in the non-linear sigma model is a non-perturbative phenomenon which is very hard to describe analytically. Nevertheless, some information can be deduced from simple dimensional arguments. The only dimensionful parameter of the non-linear sigma model is the pion decay constant. Consequently, the critical temperature should be proportional to the pion decay constant with some numerical coefficient:

$$
T_{c} \propto F_{\pi} .
$$


Here $F_{\pi}$ stands for the "bare" pion decay constant, that is, the coefficient in front of the kinetic term in the chiral Lagrangian obtained after integrating out all heavy degrees of freedom. Taking into account thermal pion loops, which effectively reduce $F_{\pi}$ [16], would be a double counting. Once the dependence of the decay constant on the temperature and the chemical potential is known, the condition (3) can be used to locate the critical line in the $T-\mu$ plane:

$$
\frac{F_{\pi}^{2}\left(T_{c}(\mu), \mu\right)}{T_{c}^{2}(\mu)}=\frac{F_{\pi}^{2}\left(T_{c}(0), 0\right)}{T_{c}^{2}(0)} .
$$

I will calculate $F_{\pi}$ as a function of temperature and chemical potential in the framework of semi-phenomenological constituent quark model of Ref. [17, 18], which has been rather successful in describing chiral dynamics and the nucleon properties [19, 18]. Pions arise in this model as chiral phases of the constituent quark mass:

$$
\mathcal{L}=\bar{\psi}\left(i \not \partial-M \mathrm{e}^{i \gamma^{5} \lambda^{a} \pi^{a}}\right) \psi=\bar{\psi}\left[i \not \partial-M\left(\frac{1-\gamma^{5}}{2} U+\frac{1+\gamma^{5}}{2} U^{\dagger}\right)\right] \psi .
$$

This form of constituent quark Lagrangian was motivated by the instanton liquid model [17]. In fact, such an interaction of constituent quarks with pions will arise after HubbardStratonovich transformation from any four-quark interaction, local or non-local, which respects the symmetries of QCD. The chiral Lagrangian in this model is obtained after integration over quark fields and subsequent derivative expansion of the fermion determinant. The pion decay constant is the coefficient before the first term with lowest number of derivatives. The expression for pion decay constant obtained in this way [19, 18] can be easily generalized to the case of non-zero temperature and chemical potential:

$$
\begin{aligned}
F_{\pi}^{2}(T, \mu) & =4 N_{c} M^{2} T \sum_{n} \int \frac{d^{3} p}{(2 \pi)^{3}} \frac{1}{\left\{[(2 n-1) \pi T-i \mu]^{2}+p^{2}+M^{2}\right\}^{2}} \\
& =F_{\pi}^{2}-\frac{N_{c} M^{2}}{2 \pi^{2}} \int_{M}^{\infty} \frac{d \omega}{\sqrt{\omega^{2}-M^{2}}}\left[\frac{1}{\mathrm{e}^{(\omega-\mu) / T}+1}+\frac{1}{\mathrm{e}^{(\omega+\mu) / T}+1}\right]
\end{aligned}
$$

where $N_{c}=3$ is the number of colors. Both the temperature and the chemical potential tend to decrease $F_{\pi}$, so larger chemical potential require lower critical temperature to satisfy Eq. (丰), as expected.

Lattice simulations give $T_{c}(0)=150 \mathrm{Mev}$ at $N_{f}=3$ and $T_{c}(0)=170 \mathrm{Mev}$ at $N_{f}=2$ in the chiral limit [15]. The boundary of the hadron phase in Fig. 1 is obtained by solving Eq. (4) with $T_{c}(0)=160 \mathrm{Mev}, M=350 \mathrm{Mev}$, and $F_{\pi}=93 \mathrm{Mev}$. Other lines in Fig. 1 are drawn somewhat arbitrarily. Since the critical temperature changes slowly in a rather wide range of chemical potentials and is always smaller than the constituent quark mass, Boltzmann statistics should be a good approximation unless $\mu$ is close to $M$. The expansion in $T / M$ then yields the following analytic expression for the critical line at $\Delta T \equiv T_{c}(\mu)-T_{c}(0) \ll T_{c}(0)$ :

$$
\frac{\Delta T}{T_{0}} \approx \frac{N_{c} M^{2}}{2 \pi F_{\pi}^{2}} \sqrt{\frac{T_{0}}{2 \pi M}} \mathrm{e}^{-M / T_{0}}\left(\cosh \frac{\mu}{T_{0}}-1\right)
$$

where $T_{0} \equiv T_{c}(0)$. 
When $T$ is small and $\mu$ is sufficiently large, the transition to the color superconducting state is expected to occur [20]. This transition cannot be driven by pion disordering, so the condition (4) is expected to work only for $\mu<M$. Nevertheless, the equation $F_{\pi}\left(0, \mu_{c}\right)=0$, to which (4) reduces at zero temperature, gives a reasonable value of $\mu_{c}$ which is roughly consistent with various estimates of a critical chemical potential for color superconducting phase transition [21].

The chiral symmetry is broken in a color superconductor by the diquark condensate whose phase excitations are similar to ordinary pions [22] and are massless in the chiral limit. However, the pion decay constant in a color superconductor is rather large [23] and it is very unlikely that pion decoherence can emerge in the color superconducting phase.

Finally, I should mention that the pseudogap phase, if exists at $N_{c}=3$, disappears in the large- $N_{c}$ limitw. The pion decay constant (chiral phase stiffness) grows as $F_{\pi}^{2}=O\left(N_{c}\right)$ at large $N_{c}$ and suppresses fluctuations of the chiral field that could drive pion decoherence. The condition (3) then gives $T_{c}=O\left(\sqrt{N_{c}}\right)$, which is smaller than an estimate based on the ideal gas approximation for baryons [12]: $T_{c}=O\left(N_{c} / \ln N_{c}\right)$, but still grows with $N_{c}$, unlike the temperature at which chiral condensate completely melts, which is supposed to be finite at $N_{c} \rightarrow \infty$.

I am grateful to M. Franz for very helpful discussions of the pseudogap phase in high- $T_{c}$ superconductors and to E. Babaev and A. Zhitnitsky for interesting comments. This work was supported by NSERC of Canada, by Pacific Institute for the Mathematical Sciences and in part by RFBR grant 01-01-00549 and RFBR grant 00-15-96557 for the promotion of scientific schools.

\section{References}

[1] H. Kleinert and E. Babaev, Phys. Lett. B 438, 311 (1998) hep-th/9809112; E. Babaev, Phys. Lett. B 497, 323 (2001) hep-th/9907089.

[2] E. Babaev, Int. J. Mod. Phys. A 16, 1175 (2001) hep-th/9909052.

[3] R. MacKenzie, P. K. Panigrahi and S. Sakhi, Int. J. Mod. Phys. A 9, 3603 (1994) hep-th/9309047.

[4] E. Babaev, Phys. Rev. D 62, 074020 (2000) hep-ph/0006087.

[5] V. J. Emery and S.A. Kivelson, Nature 374, 434 (1995).

[6] J. Corson, R. Mallozzi, J. Orenstein, J. N. Eckstein and I. Bozovic, Nature 398, 221 (1999); Z. A. Xu, N. P. Ong, Y. Wang, T. Kakeshita, S. Uchida, Nature 406, 486 (2000).

[7] V. L. Berezinsky, Sov. Phys. JETP 32, 493 (1971) [Zh. Exp. Teor. Fiz. 59, 907 (1970)]; J. M. Kosterlitz and D. J. Thouless, J. Phys. CC 6, 1181 (1973).

${ }^{*}$ Refs. [1, 2] contain a detailed discussion of what happens to the pseudogap phase in the large- $N$ limit of $(2+\varepsilon)$-dimensional Gross-Neveu model 
[8] J. B. Kogut, M. Snow and M. Stone, Nucl. Phys. B 200, 211 (1982); P. Dreher, Phys. Lett. B 281, 127 (1992); Nucl. Phys. B 389, 265 (1993).

[9] C. DeTar, Phys. Rev. D 42, 224 (1990).

[10] A. Bochkarev and J. Kapusta, Phys. Rev. D 54, 4066 (1996) hep-ph/9602405.

[11] C. DeTar and S. Dong, Phys. Rev. D 45, 4681 (1992).

[12] I. I. Kogan, A. Kovner and B. Tekin, Phys. Rev. D63, 116007 (2001) hep-ph/0101040.

[13] T. H. R. Skyrme, Nucl. Phys. 31, 556 (1962); E. Witten, Nucl. Phys. B 223, 433 (1983).

[14] R. D. Pisarski and F. Wilczek, Phys. Rev. D 29, 338 (1984); F. Wilczek, Int. J. Mod. Phys. A 7, 3911 (1992) [Erratum: A 7, 6951 (1992)].

[15] F. Karsch, E. Laermann and A. Peikert, hep-lat/0012023; F. Karsch, hep-ph/0103314.

[16] J. Gasser and H. Leutwyler, Phys. Lett. B 184, 83 (1987); S. Jeon and J. Kapusta, Phys. Rev. D 54, 6475 (1996) hep-ph/9602400.

[17] D. Diakonov and V. Y. Petrov, Nucl. Phys. B 272, 457 (1986).

[18] D. Diakonov, hep-ph/9802298.

[19] D. Diakonov, V. Y. Petrov and P. V. Pobylitsa, Nucl. Phys. B 306, 809 (1988).

[20] K. Rajagopal and F. Wilczek, hep-ph/0011333.

[21] J. Berges and K. Rajagopal, Nucl. Phys. B 538, 215 (1999) hep-ph/9804233; G. W. Carter and D. Diakonov, Phys. Rev. D 60, 016004 (1999) hep-ph/9812445; R. Rapp, T. Schafer, E. V. Shuryak and M. Velkovsky, Annals Phys. 280, 35 (2000) hep-ph/9904353.

[22] M. Alford, K. Rajagopal and F. Wilczek, Nucl. Phys. B 537, 443 (1999) hepph/9804403; T. Schafer and F. Wilczek, Phys. Rev. Lett. 82, 3956 (1999) hepph/9811473].

[23] D. T. Son and M. A. Stephanov, Phys. Rev. D 61, 074012 (2000) hep-ph/9910491 [Erratum: D 62, 059902 (2000) [hep-ph/0004095]; K. Zarembo, Phys. Rev. D 62, 054003 (2000) hep-ph/0002123; S. R. Beane, P. F. Bedaque and M. J. Savage, Phys. Lett. B 483, 131 (2000) hep-ph/0002209; V. A. Miransky, I. A. Shovkovy and L. C. Wijewardhana, Phys. Rev. D 62, 085025 (2000) hep-ph/0009129; D 63, 056005 (2001) [hep-ph/0009173. 